\title{
Kayles on special classes of graphs - An application of Sprague-Grundy theory *
}

\author{
Hans L. Bodlaender \\ Department of Computer Science, Utrecht University \\ P.O. Box 80.089, 3508 TB Utrecht, the Netherlands
}

\begin{abstract}
Kayles is the game, where two players alternately choose a vertex that has not been chosen before nor is adjacent to an already chosen vertex from a given graph. The last player that choses a vertex wins the game. We show, with help of Sprague-Grundy theory, that the problem to determine which player has a winning strategy for a given graph, can be solved in $O\left(n^{3}\right)$ time on interval graphs, on circular arc graphs, on permutation graphs, and on co-comparability graphs and in $O\left(n^{1.631}\right)$ time on cographs. For general graphs, the problem is known to be PSPACE-complete, but can be solved in time polynomial in the number of isolatable sets of vertices of the graph.
\end{abstract}

\section{Introduction}

For various reasons, games keep attracting the interest of researchers in mathematics and computer science. Games can provide for models, for instance for human thought processes, economic behavior, fault tolerance in computer systems, and computational complexity of machine models. Also, the analysis of games can provide for entertainment, or beautiful theory that is interesting on its own. It may be interesting to note that one of the first books written on graph theory [8] already contained a section on the relations between graphs and games.

In this paper we consider a combinatorial game, that is played on graphs, called Kayles. In this game, two players alternately choose a vertex from a given graph. Players may not choose a vertex that has been chosen before, and may also not choose a vertex that is adjacent to a vertex that has been chosen before. The last player that is able to choose a vertex wins the game.

*This work was partially supported by the ESPRIT Basic Research Actions of the EC under contract 7141 (project ALCOM II). 
The game can also be described as follows: when a player chooses a vertex, this vertex and all its neighbors are removed from the graph. The first player that ends his move with the empty graph wins the game.

We consider the problem: given a graph $G=(V, E)$, does there exist a winning strategy for the first player when Kayles is played on $G$ ? We denote this problem also by the name Kayles. Kayles has been shown to be PSPACE-complete by Schaefer [9].

Despite its intractability for general graphs, Kayles has some nice characteristics, which together allow for efficient algorithms that solve some special cases. We remark that the game Kayles is:

- a two player game.

- finite. The game always ends after a finite number of moves, and each player can choose each time from a finite number of possible moves.

- full-information. There is no information that is hidden to one or both players, like for instance in bridge, where cards of other players are unknown.

- deterministic. Every move gives rise to a unique position; no randomization devices (such as dice) are used.

- impartial. This means that positions have no preference towards players. In other words, for each position, either the player that must move has a winning strategy, or the other player has - this is regardless of whether player 1 or player 2 must move from the position. For example, chess is not impartial, as there are white and black pieces owned by the players.

- with 'last player that moves wins the game' rule.

These six characteristics of Kayles make it possible to analyse the game with help of Sprague-Grundy theory. Some readers may know this theory as the theory of the game Nim. In this theory, one associates to each position a (natural) number, here called nimber after [1]. (The position has nimber $i$, when it can be represented by a stack of corresponding height in the game Nim.) It is possible to do some calculations with these nimbers, and determine which player has a winning strategy. In many cases, these calculations will be intractable, but - as will be shown in this paper - in some cases, they are not.

Those basic notions and results of Sprague-Grundy theory that are needed for this paper are reviewed in section 2. For more background, we recommend the reader to consult [1] or [3]. Some graph theoretic definitions are also given in section 2 .

In section 3, we give a data structure, needed for the algorithm, described in section 4 . This algorithm solves Kayles on a graph with $n$ vertices, $e$ edges, and $\alpha$ different isolatable sets of vertices, in time $O(\alpha n e)$. In sections 5,6 and 7 we give 
modifications of this algorithm, that solve Kayles on interval graphs, circular arc graphs, cographs, permutation graphs and cocomparability graphs in polynomial time. For the result on cographs, we show that the nimber of a cograph with $n$ vertices is of size $O\left(n^{0.631}\right)$. Some final remarks are made in section 8 .

\section{Definitions and preliminary results}

In this section we give some definitions, and review some results from SpragueGrundy theory. All graphs in this paper are considered to be finite, undirected and simple. For a graph $G=(V, E)$, and a subset of the vertices $W \subseteq V$, the subgraph of $G$, induced by $W$, is denoted by $G[W]=(W,\{(v, w) \in E \mid v, w \in$ $W\}$ ). We denote $|V|$ by $n$, and $|E|$ by $e$. For $v \in V$, denote the set containing $v$ and all neighbors of $v$ by $N(v)=\{v\} \cup\{w \in V \mid(v, w) \in E\}$. For $X \subseteq V$, write $N(X)=\cup_{v \in X} N(v)$.

Definition 2.1 Let $G=(V, E)$ be a graph. A set of vertices $W \subseteq V$ is called isolatable, if there exists a set $X \subset V$, such that

1. $X$ is an independent set of vertices $(\forall v, w \in X: v \neq w \Rightarrow(v, w) \notin E)$

2. $G[W]$ is a connected component of the graph $G[V \Leftrightarrow N(X)]$.

In other words, a set $W \subseteq V$ is isolatable, if it induces a connected subgraph of $G$, and there exists an independent set of vertices $X$, such that if we remove $X$ and all neighbors of vertices in $X$ from $V$, then $W$ is one of the connected components.

In section 4 we will prove that Kayles can be solved in time polynomial in the number of different isolatable subsets of vertices of the input graph $G$. Thus, we are interested in classes of graphs where the number of different isolatable subsets is bounded by a polynomial in the number of vertices of the graph. Examples of such classes are the interval graphs, the circular arc graphs, the cographs, the permutation graphs, and the co-comparability graphs.

Definition 2.2 A graph $G=(V, E)$ is an interval graph, iff one can associate with each vertex $v \in V$ an interval on the real line $\left[b_{v}, e_{v}\right] \subseteq R$, such that for all $v, w \in V, v \neq w:(v, w) \in E \Leftrightarrow\left[b_{v}, e_{v}\right] \cap\left[b_{w}, e_{w}\right] \neq \emptyset$.

Interval graphs can be recognized in $O(n+e)$ time, and in the same order of time, the corresponding interval model can be built [2]. As only the order of the endpoints of the intervals matters, one can assume that all $b_{v}, e_{v} \in\{1,2, \ldots, 2 n\}$.

A generalization of the interval graphs are the circular arc graphs. They can be defined as follows: 
Definition 2.3 A graph $G=(V, E)$ is a circular arc graph, iff one can associate with each vertex $v \in V$ an set of integers $S_{v} \subseteq\{1,2, \ldots, 2 n\},(n=|V|)$, with $S_{v}$ either of the form $\left\{b_{v}, b_{v}+1, \ldots, e_{v} \Leftrightarrow 1, e_{v}\right\}$, or of the form $\left\{b_{v}, b_{v}+1, \ldots, 2 n \Leftrightarrow\right.$ $1,2 n\} \cup\left\{1,2, \ldots, e_{v} \Leftrightarrow 1, e_{v}\right\},\left(b_{v}, e_{v} \in\{1,2, \ldots, 2 n \Leftrightarrow 1,2 n\}\right.$,) such that for all $v, w \in V, v \neq w:(v, w) \in E \Leftrightarrow S_{v} \cap S_{w} \neq \emptyset$.

Recognition of circular arc graphs, and building the corresponding representation can be done in $O\left(n^{3}\right)$ time [10].

Definition 2.4 Let $G_{1}=\left(V_{1}, E_{1}\right)$ and $G_{2}=\left(V_{2}, E_{2}\right)$ be two disjoint graphs. The (disjoint) union of $G_{1}$ and $G_{2}$ is the graph $G_{1} \cup G_{2}=\left(V_{1} \cup V_{2}, E_{1} \cup E_{2}\right)$. The product of $G_{1}$ and $G_{2}$ is the graph $G_{1} \times G_{2}=\left(V_{1} \cup V_{2}, E_{1} \cup E_{2} \cup\{(v, w) \mid v \in\right.$ $\left.\left.V_{1}, w \in V_{2}\right\}\right)$.

In some cases, we write $G_{1} \cup G_{2}$, or $G_{1} \times G_{2}$, for graphs $G_{1}, G_{2}$ which are not disjoint. As we always want the operations $\cup$ and $\times$ to work on disjoint graphs, we assume implicitly in these cases, that we take the disjoint union or product of two disjoint graphs that are isomorphic with $G_{1}$, and $G_{2}$, respectively.

Definition 2.5 A graph is a cograph, if and only if it can be formed by the following rules:

1. Every graph with one vertex and no edges is a cograph.

2. If $G_{1}=\left(V_{1}, E_{1}\right)$ and $G_{2}=\left(V_{2}, E_{2}\right)$ are disjoint cographs, then $G_{1} \cup G_{2}$ and $G_{1} \times G_{2}$ are cographs.

To each cograph $G$, one can associate a labeled rooted tree $T_{G}$, called the cotree of $G$. Each leaf node of $T_{G}$ corresponds to a (unique) vertex of $V$. Each internal node is labeled with either a 0 or a 1 . Children of nodes labeled with 1 are labeled with 0 , and vice versa. Two vertices are connected, if and only of their lowest common ancestor in the cotree is labeled with a 1. It is possible to associate a cotree with each node of the tree. Leaf nodes correspond to the cotree with the one vertex they represent. Internal nodes labeled with 0 (1) correspond to the disjoint union (product) of the cographs, corresponding to the children of the node. $G$ equals the cograph corresponding with the root of $T_{G}$. Cographs can be recognized in $O(n+e)$ time, and in the same time the corresponding cotree can be built [4].

Definition 2.6 A graph $G=(V, E)$ is a permutation graph, iff there exist a bijection $f: V \rightarrow\{1,2, \ldots, n\}$, and a permutation $\pi:\{1,2, \ldots, n\} \rightarrow\{1,2, \ldots, n\}$, such that for all $v, w \in V:(v, w) \in E \Leftrightarrow(f(v)<f(w)$ and $\pi(f(v))>\pi(f(w)))$ or $(f(v)>f(w)$ and $\pi(f(v))<\pi(f(w)))$. 
Permutation graphs can be recognized in $O\left(n^{3}\right)$ time, and in the same time, the corresponding numbering of the vertices and permutation $\pi$ can be found [6].

A graph is a co-comparability graph, if it is the complement of a comparability graph. A graph $G=(V, E)$ is a comparability graph, if it has a transitive orientation. Co-comparability graphs can be characterized as the intersection graphs of continous functions $f:[0,1] \rightarrow R$ : one can associate to each vertex $v \in V$ a continuous function $f_{v}:[0,1] \rightarrow R$, such that for all $v, w \in V, v \neq w$ : $(v, w) \in E \Leftrightarrow \exists x \in[0,1]: f_{v}(x)=f_{w}(x)$. See [7].

Note that some of these classes are properly contained in others: every cograph is a permutation graph, every interval graph is a circular arc graph, and every permutation graph and every interval graph is a co-comparability graph.

Next, we review some notions and results from Sprague-Grundy theory. For a good introduction to this theory, the reader is referred to [3] or the less formal and entertaining [1].

A nimber is an integer in $N=\{0,1,2, \ldots\}$. For a finite set of nimbers $S \subseteq N$, define the minimum excluded nimber of $S$ as $\operatorname{mex}(S)=\min \{i \in N \mid i \notin S\}$.

We now assume that we consider positions in a two-player game, that is finite, deterministic, full-information, impartial, with 'last player wins'-rule. (As in Kayles.)

To each position in such a game, one can associate a nimber in the following way. If no move is possible in the position (and hence the player that must move loses the game), the position gets nimber 0 . Otherwise the nimber is the minimum excluded nimber of the set of nimbers of positions that can be reached in one move.

Theorem 2.1 [1, 3] There is a winning strategy for player 1 from a position, if and only if the nimber of that position is at least 1.

Denote the nimber of a position $p$ by $n b(p)$. We next define the sum of two games. For (finite, deterministic, impartial, ...) games $\mathcal{G}_{1}, \mathcal{G}_{2}$, the sum of $\mathcal{G}_{1}$ and $\mathcal{G}_{2}, \mathcal{G}_{1}+\mathcal{G}_{2}$ is the game, where each player when moving first decides whether he wants to make a move in $\mathcal{G}_{1}$ or in $\mathcal{G}_{2}$, and then selects a move in that game. The player that makes the last move (whether it is in $\mathcal{G}_{1}$ or $\mathcal{G}_{2}$ ) wins the game $\mathcal{G}_{1}+\mathcal{G}_{2}$.

Definition 2.7 Let $i_{1}, i_{2} \in N$ be nimbers. $i_{1} \oplus i_{2}$ is the binary sum of $i_{1}$ and $i_{2}$ without carry, i.e., $i_{1} \oplus i_{2}=\sum\left\{2^{j} \mid\left(\left\lfloor i_{1} / 2^{j}\right\rfloor\right.\right.$ is odd $) \Leftrightarrow\left(\left\lfloor i_{2} / 2^{j}\right\rfloor\right.$ is even $\left.)\right\}$.

In other words, write $i_{1}$ and $i_{2}$ in binary notation. For every digit, take a 1 if either $i_{1}$ has a 1 for that digit, and $i_{2}$ has a 0 for that digit, or vice versa. For example $10 \oplus 7=(8+2) \oplus(4+2+1)=8+4+1=13$.

By $\left(p_{1}, p_{2}\right)$ we denote the position in $\mathcal{G}_{1}+\mathcal{G}_{2}$, where the position in $\mathcal{G}_{i}$ is $p_{i}$ $(i=1,2)$.

Theorem 2.2 [1, 3] Let $p_{1}$ be a position in $\mathcal{G}_{1}, p_{2}$ a position in $\mathcal{G}_{2}$. The nimber of position $\left(p_{1}, p_{2}\right)$ in $\mathcal{G}_{1}+\mathcal{G}_{2}$ equals $n b\left(\left(p_{1}, p_{2}\right)\right)=n b\left(p_{1}\right) \oplus n b\left(p_{2}\right)$. 


\section{A data structure}

In this section we describe a data structure $X$, which is needed for the algorithm in section 4. For a finite, ordered set $V$, the data structure can store subsets $W \subseteq V$ with a value $\operatorname{val}(W)$, and retrieve these values. The following operations are possible on the data structure:

- $\operatorname{store}(W, x)$, for $W \subseteq V, x \in N$.

- $\operatorname{present}(W)$. Returns true, if an operation store $(W, x)$ has been performed before for any value of $x$.

- $\operatorname{val}(W)$. Returns, if $\operatorname{present}(W)$, the value $x$ of the last operation store $(W, x)$. Undefined, if not $\operatorname{present}(W)$ holds.

We assume an ordering $<$ on $V$, and assume that testing $<$ can be done in constant time. (In our application, $V$ is the set of vertices of $G$. We just number the vertices of $G$ by $v_{1}, v_{2}, \ldots$ )

We now give a simple, recursive description of the data structure $X$. It consists of three parts:

- A boolean variable empty_present, which denotes whether $\operatorname{present}(\emptyset)$.

- An integer variable empty_val, which denotes val $(\emptyset)$, if present $(\emptyset)$, and is undefined otherwise.

- A data structure $Y$, where for every $v \in V$ such that there exists a $W \subseteq V$ with present $(W)$ and $v$ is the lowest numbered element in $W$, a pointer is stored to another data structure of type $X$, that stores all sets $W \subseteq V$ with $v$ the lowest numbered vertex in $W$.

An example of this construction is given in Figure 1.

To search for a set $W \subseteq V$ (assume $W$ is given in sorted order): if $W=\emptyset$, then empty_present and empty_val give the desired information. Otherwise, let $v$ be the smallest numbered vertex in $W$, and search for $v$ in the data structure $Y$. If $v$ does not appear in $Y$, then not present $(W)$. Otherwise, follow the pointer for $v$ in data structure $Y$ to the smaller data structure $X$, and repeat the process with $W \Leftrightarrow\{v\}$ in this data structure.

To store $(W, x)$, do the following. (Again, we assume that $W$ is given in sorted order.) If the data-structure is not yet initialized, initialize it. (Reserve memory locations for empty_present and empty_val, and do what is necessary to initialize data structure $Y$.) If $W=\emptyset$, set empty_present to true, and set empty_val to $x$. Otherwise, take the smallest element $v$ from $W$, and check whether $v$ is present in $Y$. If not, insert $v$ in $Y$, and add a pointer to a new, not yet initialized data structure of type $X$. Otherwise, find the pointer stored for $v$ in $Y$. In both cases, follow the pointer, and repeat with $W \Leftrightarrow\{v\}$ on the smaller data structure. 


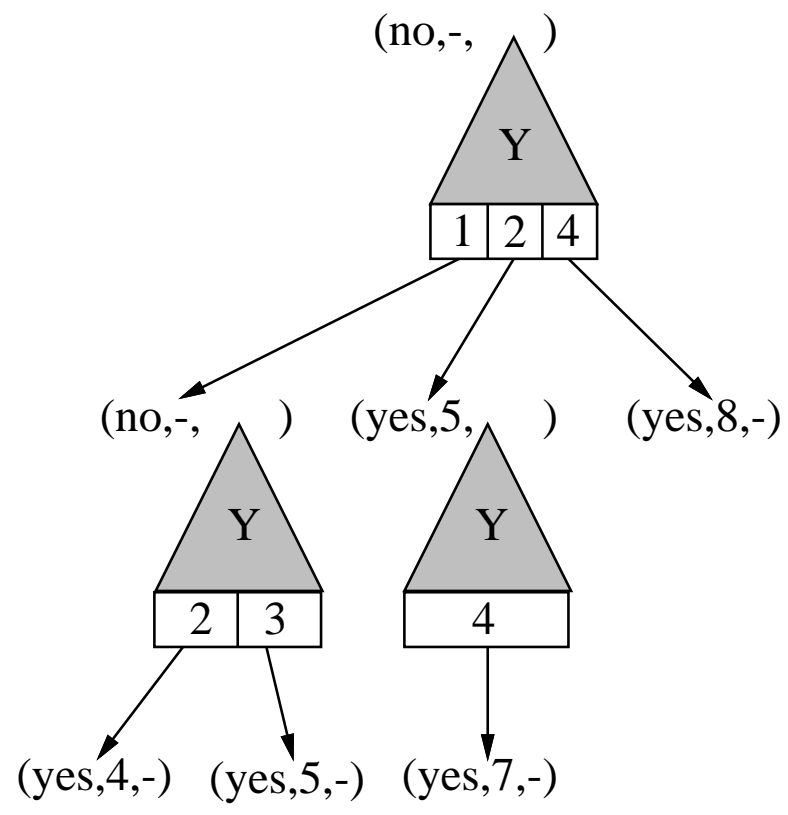

Figure 1: An example of data structure $X$, storing $\{1,2\}$ with value $4,\{1,3\}$ with value $5,\{2\}$ with value $5,\{2,4\}$ with value 7 , and $\{4\}$ with value 8

There are several possible ways to implement the data structures $Y$. Three of these possibilities are:

- as a balanced search tree, e.g. an AVL-tree. As both search and insert operations cost $O(\log n)$ time per access to a data structure of type $Y$, the total time per store, present or val operation is $O(|W| \log |V|)$. The space needed is $O\left(\sum_{\operatorname{present}(W)}|W|\right)$.

- as an van Emde Boas data structure [11]. The operations in data structure $Y$ take now $O(\log \log n)$ time and the total time per store present or val operation becomes $O(|W| \log \log |V|)$. The space needed per data structure $Y$ is $O(|V|)$, hence in total $O(|V| \alpha)$, where $\alpha$ is the number of sets $W$ that are present in the data structure.

- as a dynamic perfect hashing data structure, as described by Dietzfelbinger et al [5]. Search operations cost $O(1)$ time worst case, insert operations cost $O(1)$ expected time. Memory use is linear in the number of stored keys. This method uses randomization. Using this data structure, present and val operations cost $O(|W|)$ time worst case, and store operations cost $O(|W|)$ expected time. The amount of space that is used again is $O\left(\sum_{\text {present }(W)}|W|\right)$. 


\section{Kayles and nimbers}

As Kayles is an impartial, deterministic, finite, full-information, two-player game with the rule that the last player that moves wins the game, we can apply SpragueGrundy theory to Kayles, and we can associate with each graph $G$ the nimber of the start position of the game Kayles, played on $G$. We denote this nimber $n b(G)$, and call it the nimber of $G$.

Note that when $n b(G)$ is known, then one can directly determine, with theorem 2.2 which player has a winning strategy.

An important observation is the following: when $G=G_{1} \cup G_{2}$ for disjoint graphs $G_{1}, G_{2}$, then the game Kayles, played on $G$, is the sum of the game Kayles, played on $G_{1}$, and the game Kayles, played on $G_{2}$. Hence, by theorem 2.2, we have the following result.

Theorem $4.1 n b\left(G_{1} \cup G_{2}\right)=n b\left(G_{1}\right) \oplus n b\left(G_{2}\right)$.

The second important observation is that when some vertices $X \subseteq V$ have been chosen, then the nimber of the resulting position is equal to the nimber of $G[V \Leftrightarrow N(X)]$. In other words: the game does not change when we remove all vertices that have been chosen and all their neighbors from $G$. In particular, the nimber of the position arising from playing $v$ in $G$ as first move equals $n b(G[V \Leftrightarrow$ $N(v)]$ ). Moreover, the vertex set of each connected component of $G[V \Leftrightarrow N(X)]$ is an isolatable set.

These two basic observations make it possible to compute the nimber of graphs with a polynomial number of isolatable sets of vertices in polynomial time.

Theorem 4.2 There exists algorithms, that given a graph $G=(V, E)$, computes $n b(G)$ in $O\left(\alpha n e+\alpha n^{2} \log \log n\right)$ time worst case, or in $O(\alpha n e)$ expected time, respectively, and in $O(\alpha n)$ space, where $\alpha$ is the number of different isolatable sets of vertices of $G$.

Proof: We use a data structure $X$, as described in section 3 , in which we store isolatable sets $W \subseteq V$ with $n b(G[W])$. Initially, $X$ is empty. The following recursive procedure, when called with a set $W \subseteq V$ returns the value of $n b(G[W])$. It is called by the main algorithm with compute_nimber $(W)$, for every connected component $G[W]$ of $G$. The nimber of $G$ is the $\oplus$-sum of these nimbers.

procedure compute_nimber $(W)$ : integer;

$\{W \subseteq V$. The procedure returns the nimber $n b(G[W])$.

begin

if $\operatorname{present}(W)$ then $\operatorname{return}(\operatorname{val}(W))$

else

$M:=\emptyset ;$

for all $v \in W$ do 
begin compute the connected components of $G[W \Leftrightarrow N(v)]$.

Suppose these components have sets of vertices

$W_{1}, \ldots, W_{r} \subseteq W$.

$\left\{\right.$ We compute now: $n b(G[W \Leftrightarrow N(v)])=n b\left(G\left[W_{1}\right]\right) \oplus \ldots$

$\left.\oplus n b\left(G\left[W_{r}\right]\right) \cdot\right\}$

$n:=0$;

for $i:=1$ to $r$ do

begin $n:=n \oplus$ compute_nimber $\left(W_{i}\right)$;

end;

$\{$ Now $n=n b(G[W \Leftrightarrow N(v)])$.

$M:=M \cup\{m\}$; end;

$\{M$ forms the set of the nimbers of positions, reachable in one move from $G[W]$.

ans $:=\operatorname{mex}(M)$;

store $(W$, ans $)$;

return (ans)

end.

Correctness of the algorithm follows from the earlier made observations. For the running time of the algorithm, observe that the computations in the elsepart are carried at most once for a set $W$. compute_nimber is only called with isolatable sets $W \subseteq V$. It is possible to keep all sets $W, W_{i}$ sorted in time, linear in $W$. We count the time, needed for one call of compute_nimber $(W)$, including the calls of present $\left(W_{i}\right)$, and if $\operatorname{present}\left(W_{i}\right)$, of $\operatorname{val}\left(W_{i}\right)$. We need $O\left(n^{2}+n e\right)=O(n e)$ time for all computations of connected components. In case the data structures $Y$ are implemented with van Emde Boas data structures, the calls to present $\left(W_{i}\right)$ cost $O\left(\left|W_{i}\right| \log \log n\right)$ time each. In total, this is $O\left(n^{2} \log \log n\right)$ time. If we use dynamic perfect hashing, the expected time becomes $O\left(n^{2}\right)$. The total over all $\alpha$ isolatable sets of vertices gives the bounds stated in the theorem. Clearly, the space needed for the data structure is bounded by $O(\alpha n)$.

\section{Kayles on interval graphs and circular arc graphs}

In this section we consider Kayles when played on interval graphs, or on circular arc graphs. We show that nimbers of these graphs, and hence players with a winning strategy, can be determined quickly, as there are only $O\left(n^{2}\right)$ isolatable sets of vertices, and these have an nice and easy structure.

Assume that $G=(V, E)$ is an interval graph, and let with each vertex $v \in V$ an interval $\left[b_{v}, e_{v}\right]$ be associated, such that $b_{v}, e_{v} \in\{1,2, \ldots, 2 n\}, b_{v} \leq e_{v}$, and for all $v, w \in V, v \neq w:(v, w) \in E \Leftrightarrow\left[b_{v}, e_{v}\right] \cap\left[b_{w}, e_{w}\right] \neq \emptyset$. 
Lemma 5.1 Let $W \subseteq V$ be an isolatable set of vertices. Then there exist numbers $b_{W}, e_{W} \in\{1,2, \ldots\}$, such that for all $v \in V: v \in W \Leftrightarrow b_{W} \leq b_{v} \leq e_{v} \leq e_{W}$.

Proof: Take $b_{W}=\min \left\{b_{v} \mid v \in W\right\}$, and $e_{W}=\max \left\{e_{v} \mid v \in W\right\}$. Suppose $b_{v_{1}}=b_{W}, e_{v_{2}}=e_{W}, v_{1}, v_{2} \in W$. Clearly $v \in W \Rightarrow b_{W} \leq b_{v} \leq e_{v} \leq e_{W}$.

Suppose there exists a vertex $w \notin W$ with $b_{W} \leq b_{w} \leq e_{w} \leq e_{W}$. Either $w \in X$, or $w$ is adjacent to a vertex $x \in X$. In both cases, we have a vertex $x \in X$ with $\left[b_{x}, e_{x}\right] \cap\left[b_{W}, e_{W}\right] \neq \emptyset$. As $x$ may not be adjacent to $v_{1}$ or $v_{2}$, we have $b_{W}<b_{x} \leq e_{x}<e_{W}$. As vertices in $W$ are not adjacent to $X$, we can write $W$ as the disjoint union of $A=\left\{v \in W \mid e_{v}<b_{x}\right\}$ and $B=\left\{v \in W \mid e_{x}<b_{v}\right\}$. A and $B$ are non-empty, as they contain $v_{1}$ and $v_{2}$, respectively, and there cannot be an edge between a vertex in $A$ and a vertex in $B$. Hence $G[W]$ is not connected, contradiction.

From this result and theorem 4.2, it follows that Kayles can be solved in polynomial time on interval graphs. We give a modification of this general result, which uses $O\left(n^{3}\right)$ time.

Let $G_{i j}(1 \leq i \leq j \leq n)$ denote the subgraph of $G$, induced by all vertices $v \in V$ with $i \leq b_{v} \leq e_{v} \leq j: G_{i j}=G\left[\left\{v \in V \mid i \leq b_{v} \leq e_{v} \leq j\right\}\right]$. The algorithm uses a two-dimensional array $A$, where $A(i, j)$ will store the value of $n b\left(G_{i j}\right)$.

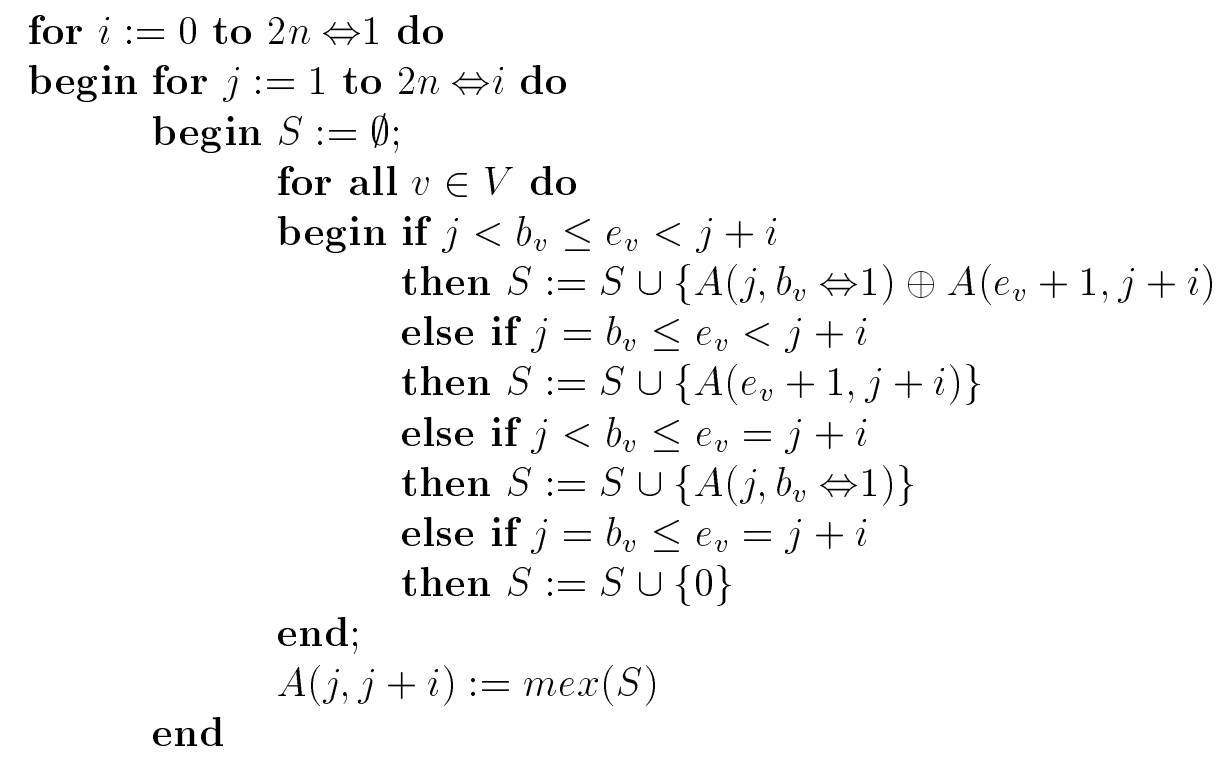

end;

output $A(1,2 n)$.

The algorithm computes $n b\left(G_{j(j+i)}\right)$ successively for $i$ ranging from 0 to $2 n \Leftrightarrow 1$ and $j$ from 1 to $2 n \Leftrightarrow i$. If $j \leq b_{v} \leq e_{v} \leq j+i$, then $v$ belongs to $G_{j(j+i)}$. Then, removing $v$ and its neighbors from $G_{j(j+i)}$ splits $G_{j(j+i)}$ in two parts $G_{j\left(b_{v}-1\right)}$ and $G_{\left(e_{v}+1\right)(j+i)}$. If either $j=b_{v}$, or $e_{v}=j+i$, then one of this parts is empty. If both $j=b_{v}$ and $e_{v}=j+i$, then both parts are empty and the resulting nimber is 
0. It follows that for each $v$ in $G_{j(j+i)}, n b\left(G_{j(j+i)} \Leftrightarrow N(v)\right)$ is computed correctly, and put into $S$. Hence $\operatorname{mex}(S)=n b\left(G_{j(j+i)}\right)$. This shows correctness of the algorithm.

Clearly, the algorithm uses $O\left(n^{3}\right)$ time. With theorem 2.1, we can directly determine which player has a winning strategy after execution of the algorithm.

Theorem 5.2 There exists an $O\left(n^{3}\right)$ algorithm for Kayles on interval graphs.

The same result holds for circular arc graphs. We use basically the same algorithm. Define $V_{i j}$ to be the set $\left\{v \in V \mid S_{v} \subseteq\{i, i+1, \ldots, j \Leftrightarrow 1, j\}\right\}$, if $i \leq j$, and $\left\{v \in V \mid S_{v} \subseteq\{i, i+1, \ldots, 2 n \Leftrightarrow 1,2 n\} \cup\{1,2, \ldots, j \Leftrightarrow 1, j\}\right.$, if $i>j$. Similar as before, we can compute all $n b\left(G_{i j}\right)$, now for all pairs $i, j$, with $i, j \in\{1,2, \ldots, 2 n\}$, using dynamic programming, in $O\left(n^{3}\right)$ time in total.

Theorem 5.3 There exists an $O\left(n^{3}\right)$ algorithm for Kayles on circular arc graphs.

\section{$6 \quad$ Kayles on cographs}

Cographs also have the property that the number of isolatable sets is bounded by a polynomial.

Theorem 6.1 Let $G=(V, E)$ be a cograph, and let $T_{G}$ be its corresponding cotree. Let $W \subseteq V$ be an isolatable set of size at least two. Then there exists a 1-labeled internal node $i$ in $T_{G}$ such that $W$ equals all vertices, represented by a leaf-descendant of $i$ in $T_{G}$.

Proof: Let $i$ be the lowest common ancestor in $T_{G}$ of all vertices in $W$. $i$ is an internal node with label 1 . (If $i$ has label 0 , then $G[W]$ is not connected.) $i$ cannot have a leaf-descendant $x$ with $x \in X$, because then $i$ will be lowest common ancestor of a vertex $w \in W$ and $x$, hence $(w, x) \in E$, contradiction.

Suppose a leaf-descendant $v$ of $i$ does not belong to $W . v$ must be adjacent to a vertex $x \in X$, and the lowest common ancestor $j$ of $v$ and $x$ must be a 1labeled internal node that is an ancestor of $i$. But now $j$ is also the lowest common ancestor of an arbitrary vertex $w \in W$ and $x$, hence $(w, x) \in E$, contradiction.

It follows that there are $O(n)$ isolatable sets in a cograph with $n$ vertices. From theorem 4.2, it follows that Kayles can be solved on cographs in $O\left(n^{2} e\right)$ expected time, or $O\left(n^{2} e+n^{3} \log \log n\right)$ worst case time. A better algorithm can be obtained with a more careful analysis. Hereto, we compute for each cograph, associated with a node in the cotree $T_{G}$, the set of nimbers of the positions, reachable in one move.

Definition 6.1 Let $G=(V, E)$ be a graph. The nimberset of $G$ is the set of nimbers $n b s(G)=\{n b(G[V \Leftrightarrow N(v)] \mid v \in V\}$. 
Recall that $n b(G)=\operatorname{mex}(n b s(G))$. We use the following notation: for a set of nimbers $S \subseteq N$, and a nimber $\alpha$, we denote $\alpha \oplus S=\{\alpha \oplus \beta \mid \beta \in S\}$.

Lemma 6.2 Let $G_{1}=\left(V_{1}, E_{1}\right), G_{2}=\left(V_{2}, E_{2}\right)$ be two disjoint graphs.

(i) $n b s\left(G_{1} \cup G_{2}\right)=n b\left(G_{2}\right) \oplus n b s\left(G_{1}\right) \cup n b\left(G_{1}\right) \oplus n b s\left(G_{2}\right)$.

(ii) $n b s\left(G_{1} \times G_{2}\right)=n b s\left(G_{1}\right) \cup n b s\left(G_{2}\right)$.

Proof: (i) $n b s\left(G_{1} \cup G_{2}\right)=\left\{n b\left(G_{1}\left[V_{1} \Leftrightarrow N(v)\right] \cup G_{2}\right) \mid v \in V_{1}\right\} \cup\left\{n b\left(G_{2}\left[V_{2} \Leftrightarrow\right.\right.\right.$ $\left.\left.N(v)] \cup G_{1}\right) \mid v \in V_{2}\right\}=n b\left(G_{2}\right) \oplus\left\{n b\left(G_{1}\left[V_{1} \Leftrightarrow N(v)\right] \mid v \in V_{1}\right\} \cup n b\left(G_{1}\right) \oplus\right.$ $\left\{n b\left(G_{2}\left[V_{2} \Leftrightarrow N(v)\right] \mid v \in V_{2}\right\}=n b\left(G_{2}\right) \oplus n b s\left(G_{1}\right) \cup n b\left(G_{1}\right) \oplus n b s\left(G_{2}\right)\right.$.

(ii) Write $G=G_{1} \times G_{2} . n b s(G)=\left\{n b\left(G\left[V_{1} \cup V_{2} \Leftrightarrow N(v)\right]\right) \mid v \in V_{1}\right\} \cup\left\{n b\left(G\left[V_{1} \cup\right.\right.\right.$ $\left.\left.\left.V_{2} \Leftrightarrow N(v)\right]\right) \mid v \in V_{2}\right\}=\left\{n b\left(G_{1}\left[V_{1} \Leftrightarrow N(v)\right]\right) \mid v \in V_{1}\right\} \cup\left\{n b\left(G_{2}\left[V_{2} \Leftrightarrow N(v)\right]\right) \mid v \in V_{2}\right\}$ $=n b s\left(G_{1}\right) \cup n b s\left(G_{2}\right)$.

The lemma can be generalized as follows.

Lemma 6.3 Let $G_{1}=\left(V_{1}, E_{1}\right), G_{2}=\left(V_{2}, E_{2}\right), \ldots, G_{r}=\left(V_{r}, E_{r}\right)$ be $r$ disjoint graphs.

(i) $n b s\left(G_{1} \cup G_{2} \cup \ldots \cup G_{r}\right)=\bigcup_{1 \leq i \leq r} n b\left(G_{1}\right) \oplus n b\left(G_{2}\right) \oplus \ldots \oplus n b\left(G_{i-1}\right) \oplus n b\left(G_{i+1}\right) \oplus$ $\ldots \oplus n b\left(G_{r}\right) \oplus n b s\left(G_{i}\right)$.

(ii) $n b s\left(G_{1} \times G_{2} \times \ldots \times G_{r}\right)=n b s\left(G_{1}\right) \cup n b s\left(G_{2}\right) \cup \ldots \cup n b s\left(G_{r}\right)$.

The idea is to use these lemmas to compute for all internal nodes in $T_{G}$, the nimber and nimberset of the corresponding cograph. It is helpful for decreasing the running time of this computation, when we know what the maximum nimber is that a cograph with $n$ vertices can attain.

Let $s(K)$ denote the minimum number of vertices of a cograph $G$ with nimber at least $2^{K}$. We will show that $s(K)=3^{K}$.

Lemma 6.4 For all $K \geq 0, s(K) \leq 3^{K}$.

Proof: We give a series of cographs, $H_{0}, H_{1}, H_{2}, \ldots$, with $H_{K}$ containing exactly $3^{K}$ vertices, and $n b s\left(H_{K}\right)=\left\{0,1,2, \ldots, 2^{K} \Leftrightarrow 1\right\}$, and hence $n b\left(H_{K}\right)=2^{K}$.

For $H_{0}$, take a graph with one vertex and no edges. For $K \geq 1$, take $H_{K}=$ $\left(H_{K-1} \cup H_{K-1}\right) \times H_{K-1}$. With lemma 6.2 , one easily verifies with induction that $H_{K}$ fulfills the conditions mentioned above.

Theorem 6.5 For all $K \geq 0, s(K)=3^{K}$.

The proof of theorem 6.5 is given in Appendix A.

Corollary 6.6 For every cograph $G=(V, E)$ with $n$ vertices, $n b(G)<2 n^{1 / \log 3}$. 
Proof: $\quad$ Take the largest $K$ with $2^{K} \leq n b(G)$. By theorem 6.5, $n \geq 3^{K}$, hence $n b(G)<2 \cdot 2^{K}=2 \cdot 3^{(1 / \log 3) K} \leq 2 \cdot n^{1 / \log 3}$.

Note that $1 / \log 3 \approx 0.63093$.

We now give an algorithm that solves Kayles on cographs. We suppose that cograph $G$ is given together with its cotree $T_{G}$. For each node $i$ in $T_{G}$, let $G_{i}$ denote the cograph corresponding with this node, and write $n b(i)=n b\left(G_{i}\right)$, and $n b s(i)=n b s\left(G_{i}\right)$. Let $z=\left\lfloor 2 n^{1 / \log 3}\right\rfloor$. To store $n b s(i)$ for each node $i$ in $T_{G}$, associate with each node $i$ in $T_{G}$ a boolean array with entries for all nimbers $0,1, \ldots, z$. The algorithm computes $n b s(i)$ and $n b(i)$ for all nodes $i$ in $T_{G}$. For easier presentation, we state the algorithm as a recursive procedure, which is called with compute_nimbersets $(r)$, with $r$ the root of $T_{G}$.

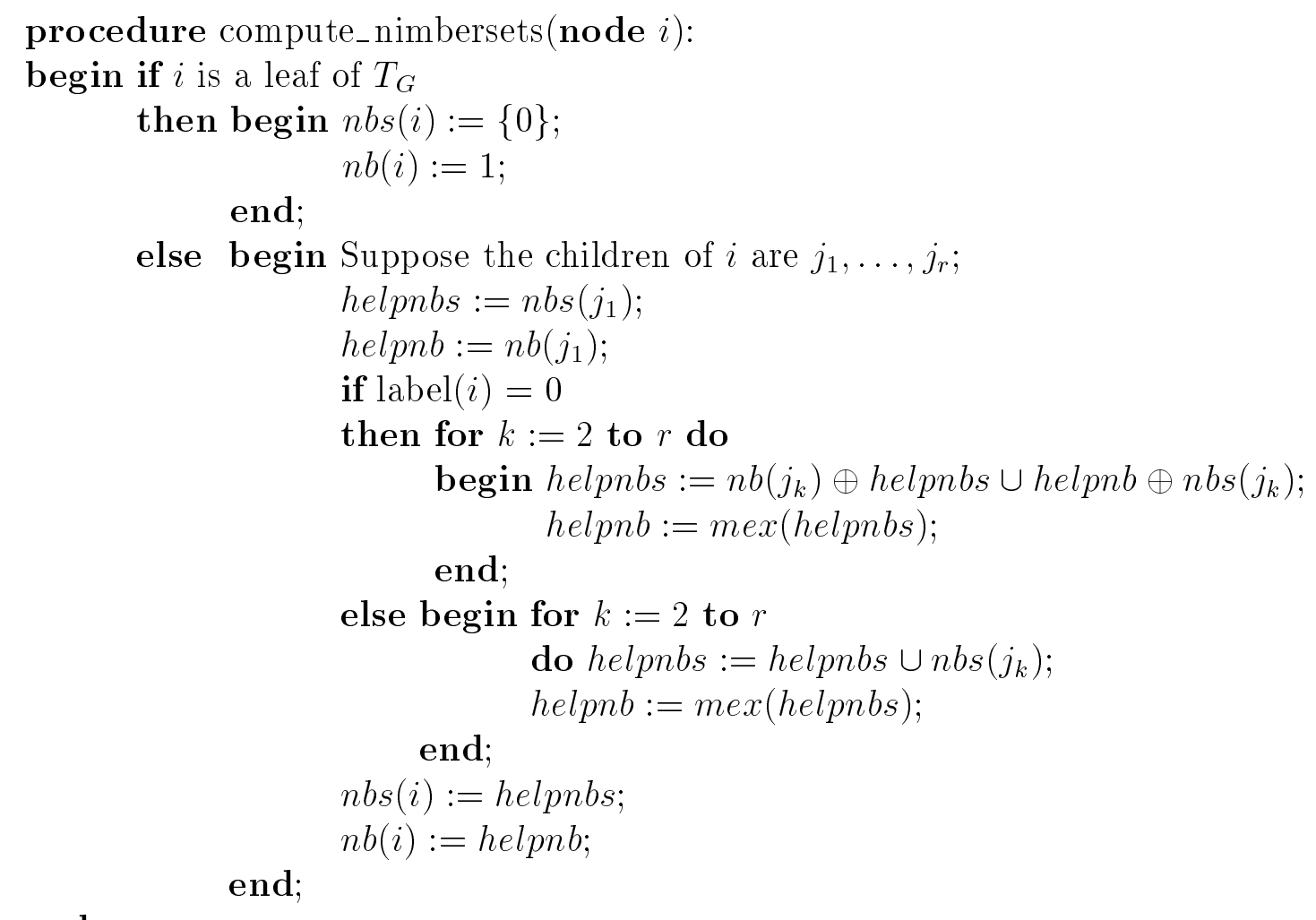

end.

Correctness follows from lemmas 6.2 and 6.3. Taking the union of two sets of nimbers, and taking the $\oplus$-sum of a nimber and a set of nimbers can be done in $O(z)$ time. As $T_{G}$ has $n$ leaves, and hence $\leq n \Leftrightarrow 1$ edges, a linear number of these operations is done. Hence, the total time of the algorithm is bounded by $O(n z)=O\left(n^{1+1 / \log 3}\right)=O\left(n^{1.631}\right)$.

Theorem 6.7 Kayles can be solved on cographs in $O\left(n^{1+1 / \log 3}\right)=O\left(n^{1.631}\right)$ time. 


\section{Kayles on permutation graphs and co- comparability graphs}

In this section we show that Kayles can be solved in $O\left(n^{3}\right)$ time on permutation graphs and co-comparability graphs. The method is more or less similar to the method used for interval graphs.

One can show that the number of isolatable subsets of a permutation graph is bounded by $O\left(n^{4}\right)$. We do not need this fact, and do not prove it here.

We suppose that we have a permutation graph $G=(V, E)$, with $V=$ $\{1,2, \ldots, n\}$, and a permutation $\pi:\{1,2, \ldots, n\} \rightarrow\{1,2, \ldots, n\}$, such that $E=\{(v, w) \mid v, w \in V,(v<w \wedge \pi(v)>\pi(w)) \vee(v>w \wedge \pi(v)<\pi(w))\}$. Further, assume $\pi(0)=0$, and $\pi(n+1)=n+1$.

For $0 \leq i \leq j \leq n+1$, denote $V_{i j}=\{v \in V \mid i<v<j$ and $\pi(i)<\pi(v)<$ $\pi(j)\}$. Note that $V_{0(n+1)}=V$, and $V_{i i}=V_{i(i+1)}=\emptyset$. Write $G_{i j}=G\left[V_{i j}\right]$.

The algorithm in this section is based on computing the nimbers $n b\left(G_{i j}\right)$, for all $i, j, 0 \leq i \leq j \leq n+1$, with dynamic programming.

Lemma 7.1 Let $v \in V_{i j}, 0 \leq i \leq j \leq n+1 . G\left[V_{i j} \Leftrightarrow N(v)\right]$ is the disjoint union of $G_{i v}$ and $G_{v j}$.

Proof: From the definitions it follows that $V_{i j} \Leftrightarrow N(v)=V_{i v} \cup V_{v j}$. Further note that $V_{i v} \cap V_{v j}=\emptyset$, and no vertex in $V_{i v}$ can be adjacent to a vertex in $V_{v j}$.

It follows that each isolatable subset is of the form $V_{i j}$ for some $i, j \in$ $\{0,1, \ldots, n+1\}$. From this lemma, and theorem 4.1, the following lemma can be derived directly:

Lemma 7.2 Let $0 \leq i \leq j \leq n+1$.

(i) For all $v \in V_{i j}: n b\left(G\left[V_{i j} \Leftrightarrow N(v)\right]\right)=n b\left(G_{i v}\right) \oplus n b\left(G_{v j}\right)$.

(ii) $n b\left(G_{i j}\right)=\operatorname{mex}\left\{n b\left(G_{i v}\right) \oplus n b\left(G_{v j}\right) \mid v \in V_{i j}\right\}$.

This lemma suggests the following dynamic programming algorithm.

for $i:=0$ to $n+1$ do $n b\left(G_{i i}\right):=0$;

for $i:=0$ to $n$ do $n b\left(G_{i(i+1)}\right):=0$;

for $i:=2$ to $n+1$ do

begin for $j:=0$ to $n+1$ do

begin $S:=\emptyset$;

for all $v \in V$ with $j<v<j+i$ and $\pi(j)<\pi(v)<\pi(j+i)$

do $S:=S \cup\left\{n b\left(G_{j v}\right) \oplus n b\left(G_{v(j+i)}\right)\right\}$;

end

$n b\left(G_{j(j+i)}\right):=\operatorname{mex}(S)$;

end;

output $n b\left(G_{0(n+1)}\right)$. 
Again, by theorem 2.1, player 1 has a winning strategy, if and only if $n b\left(G_{0(n+1)}\right)>0$. The algorithm clearly takes $O\left(n^{3}\right)$ time.

Theorem 7.3 Kayles can be solved in $O\left(n^{3}\right)$ time on permutation graphs.

In a similar manner, Kayles can be solved in $O\left(n^{3}\right)$ time on co-comparability graphs. Suppose $G=(V, E)$ is a co-comparability graph. Suppose $V=$ $\{1,2, \ldots, n\}$, with this ordering on the vertices a topological ordering of a transitive orientation of the complement of $G$. Such an orientation can be found in $O(d \cdot e)$ time, where $d$ is the maximum degree of a vertex (see [6], p.129). There exists a collection of continuous functions $\left\{f_{v}:[0,1] \rightarrow R \mid v \in V\right\}$, such that these form an intersection model of $G$ (i.e., for all $v, w \in V, v \neq w$ : $\left.(v, w) \in E \Leftrightarrow \exists x \in[0,1]: f_{v}(x)=f_{w}(x)\right)$, and for all $v, w \in V:$ if for all $x \in[0,1]:$ $f_{v}(x)<f_{w}(x)$, then $v<w[7]$. Our algorithm does not construct these functions; we use them only for showing correctness of our algorithm.

We have two additional functions: define $f_{0}:[0,1] \rightarrow R$ by $f_{0}(x)=$ $\min _{v \in V} f_{v}(x) \Leftrightarrow 1$, and define $f_{n+1}:[0,1] \rightarrow R$ by $f_{n+1}(x)=\max _{v \in V} f_{v}(x)+1$. Note that $f_{0}$ is strictly smaller than all other functions $f_{v}$, and $f_{n+1}$ is strictly larger than all these functions. Write $v \prec w$, iff for all $x \in[0,1]: f_{v}(x)<f_{w}(x)$.

For $0 \leq i \leq j \leq n+1$, write $V_{i j}=\left\{v \in V \mid i \prec v \prec j\right.$. Write $G_{i j}=G\left[V_{i j}\right]$. Again, for $v \in V_{i j}, 0 \leq i \leq j \leq n+1$, it holds that $G\left[V_{i j} \Leftrightarrow N(v)\right]$ is the disjoint union of $G_{i v}$ and $G_{v j}$. Testing whether a vertex $v$ with $i<v<j$ belongs to $V_{i j}$ can be done easily: $v \in V_{i j} \Leftrightarrow(i, v) \notin E$ and $(j, v) \notin E$. (Note that either $v \prec i$ and hence $v<i$, or $i \prec v \prec j$, or $j \prec v$ and hence $j<v$, or $f_{v}$ intersects with $f_{i}$ or with $f_{j}$ and hence $(i, v) \in E$ or $(j, v) \in E$.) Thus, we can use almost the same dynamic programming algorithm as we have used for permutation graphs: replace the sixth line of the algorithm given above by:

for all $v \in V$ with $i<v<j$ and $(i, v) \notin E$ and $(j, v) \notin E$

Correctness of the algorithm follows similarly as in earlier cases. Again, each isolatable subset is of the form $V_{i j}$ for some $i, j \in\{0,1, \ldots, n+1\}$.

Theorem 7.4 Kayles can be solved in $O\left(n^{3}\right)$ time on co-comparability graphs.

\section{Final remarks}

In this paper, we obtained polynomial time algorithms for Kayles, when restricted to several well studied classes of graphs. For several other interesting classes of graphs, the complexity of Kayles is still open. Probably the most notable of these classes is the class of trees. Already in 1978, Schaefer mentioned as an open problem the complexity of Kayles, when restricted to trees where only one vertex has degree at least three [9]. To the author's best knowledge, this problem is still unresolved. 


\section{A Proof of theorem 6.5}

Here we show that for all $K \geq 0, s(K) \geq 3^{K}$. As it is shown in lemma 6.4 that $s(K) \leq 3^{K}$, theorem 6.5 follows.

First observe that $s(0)=1$, as the empty graph has nimber 0 , and a graph with one vertex has nimber 1 . The graph with two vertices and no edges has nimber 0 , and the graph with two vertices and one edge has nimber 1 . Hence $s(1) \geq 3$. We will now show that for all $K \geq 1, s(K+1) \geq 3 \cdot s(K)$. With induction, the result then follows.

Suppose $G=(V, E)$ is a cograph with nimber $\geq 2^{K+1}$, with minimum size of $n=|V|$. Let $T_{G}$ be the corresponding cotree. We write $G_{i}$ for the cograph, corresponding with node $i$ in $T_{G}$.

First observe, that if for any node $i$ in $T_{G}, 2^{K+1} \in n b s\left(G_{i}\right)$, then $G_{i}$ contains a subgraph with nimber $2^{K+1}$. The size of this subgraph is smaller than the size of $G$, hence $G$ was not of minimum size. Thus we may assume that $n b s(G)=$ $\left\{0,1,2, \ldots, 2^{K+1} \Leftrightarrow 1\right\}$, and for all $i \in I: n b s\left(G_{i}\right) \subseteq\left\{0,1,2, \ldots, 2^{K+1} \Leftrightarrow 1\right\}$.

We say that a node $i \in T_{G}$ is $K$-heavy, if $n b\left(G_{i}\right) \geq 2^{K}$ or $n b s\left(G_{i}\right) \cap\left\{2^{K}, 2^{K}+\right.$ $\left.1, \ldots, 2^{K+1} \Leftrightarrow 1\right\} \neq \emptyset$. A node $i \in T_{G}$ is $K$-precise, if $n b s\left(G_{i}\right)=\left\{0,1,2, \ldots, 2^{K} \Leftrightarrow 1\right.$. Note that for a $K$-precise node $i, n b\left(G_{i}\right)=2^{K}$.

Claim A.1 If $i_{0}$ is $K$-heavy, then $i_{0}$ is $K$-precise, or $i_{0}$ has a descendant that is $K$-precise.

Proof: From lemma 6.3 it easily follows that every $K$-heavy node either is $K$-precise, or has a $K$-heavy child.

As the root $r$ of $T_{G}$ is $K$-heavy, it follows that there must be at least one $K$ precise node in $T_{G}$. Note that if a $K$-precise node $i$ has a descendant $j \neq i$ that is also a $K$-precise node, then $G$ is not minimal: use the cograph corresponding to the cotree, obtained by replacing the subtree rooted at $i$ in $T_{G}$ by the subtree rooted at $j$. So assume no $K$-precise node has a descendant which is also $K$ precise.

Claim A.2 There are at least two K-precise nodes in $T_{G}$.

Proof: Suppose that $i$ is the only $K$-precise node in $T_{G}$. Then the only $K$ heavy nodes in $T_{G}$ are the nodes on the path from $T_{G}$ to root $r$. With induction, one can prove that for each node $j$ on this path, $\left\{0,1,2, \ldots, 2^{K} \Leftrightarrow 1\right\} \subseteq n b s\left(G_{j}\right)$. (Use lemma 6.3, and note that only one term contains the binary factor $2^{K}$.) Hence, if a predecessor $j_{0}$ of $i$ is 1-labeled, it follows that for the unique $K$ heavy child $j_{1}$ of $j_{0}, n b s\left(G_{j_{1}}\right)=n b s\left(G_{j_{0}}\right)$. Hence $G$ was not of minimum size, contradiction.

So we may assume that $i$ has exactly one predecessor, namely $r$, which is labeled with a 0 . Hence we can write $G=G_{i} \cup H$. ( $H$ is the union of all 
cographs, corresponding to the other childs of $r$.) $n b s\left(G_{i}\right)=\left\{0,1,2, \ldots, 2^{K} \Leftrightarrow 1\right\}$, $n b s(H) \subseteq\left\{0,1,2, \ldots, 2^{K} \Leftrightarrow 1\right\}$. If $n b(H)<2^{K}$, then $2^{K}+n b(H) \notin n b s\left(G_{i} \cup H\right)$, hence $n b(G)<2^{K+1}$. So $n b(H)=2^{K}$. Applying lemma 6.2 it follows that $n b s\left(G_{i} \cup H\right)=\left\{2^{K}, 2^{K}+1,2^{K}+2, \ldots, 2^{K+1} \Leftrightarrow 1\right\}$, contradiction.

If there are at least three $K$-precise nodes, then note that each of these must have at least $s(K)$ leaf-descendants. Hence $s(K+1) \geq 3 \cdot s(K)$. Assume now there are exactly two $K$-precise nodes, $i_{0}$ and $i_{1}$. Let $i_{2}$ be the lowest common ancestor of $i_{0}$ and $i_{1}$. Similar as above, we can argue that $G$ is not of minimum size, if a node between $i_{0}$ and $i_{2}$ or a node between $i_{1}$ and $i_{2}$ has a label 1 , and if $i_{2}$ has label 1 , then it has exactly two children, which are both $K$-heavy. (Each subtree rooted at one of these two children contains exactly one of $i_{0}$ and $i_{1}$, in this case.)

We consider now two cases, namely that $i_{2}$ has label 0 , and that $i_{2}$ has label 1.

Case 1. $i_{2}$ has label 0. Then $G_{i_{2}}$ can be written as $G_{i_{2}}=G_{i_{0}} \cup G_{i_{1}} \cup G_{j_{1}} \cup$ $\ldots G_{j_{r}}$. Write $H=G_{j_{1}} \cup \ldots \cup G_{j_{r}}$. Note that $n b s(H) \subseteq\left\{0,1,2, \ldots, 2^{K} \Leftrightarrow 1\right\}$, as none of the nodes $j_{1}, \ldots, j_{r}$ is $K$-heavy. If $n b(H)=2^{K}$, then $n b s\left(G_{i_{2}}\right)=$ $\left\{0,1, \ldots, 2^{K} \Leftrightarrow 1\right\}$ and $i_{2}$ is $K$-precise, contradiction. So $n b(H)<2^{K}$, and it follows that $n b s\left(G_{i_{2}}\right)=\left\{2^{K}, 2^{K}+1, \ldots, 2^{K+1} \Leftrightarrow 1\right\} \cup n b s(H)$.

Claim A.3 Let $j$ be a node in $T_{G}$ on the path from $i_{2}$ to $r$. Let $H_{j}$ be the subgraph of $G_{j}$, obtained by removing all leaf-descendants of $i_{0}$ and of $i_{1}$ from $G_{j}$. Then $n b s\left(G_{j}\right)=n b s\left(H_{j}\right) \cup\left\{2^{K}, 2^{K}+1, \ldots, 2^{K+1} \Leftrightarrow 1\right\}$.

Proof: With induction. For $j=i_{2}$, the claim holds, as is argued above. Suppose the claim holds for the $K$-heavy child $j^{\prime}$ of $j$. Note that none of the other children of $j$ is $K$-heavy. We must have that $n b\left(G_{j^{\prime}}\right)=n b\left(H_{j^{\prime}}\right)<2^{K}$, otherwise $n b\left(G_{j^{\prime}}\right)=2^{K+1}$, which contradicts the minimality of $G$.

Write $G_{j}=G_{j^{\prime}} \cup H$, or $G_{j}=G_{j^{\prime}} \times H$. If $j$ is labeled with a 0 , then $H_{j}=$ $H_{j^{\prime}} \cup H$, and $n b s\left(G_{j}\right)=n b\left(G_{j^{\prime}}\right) \oplus n b s(H) \cup n b(H) \oplus n b s\left(H_{j^{\prime}}\right) \cup n b(H) \oplus\left\{2^{K}, 2^{K}+\right.$ $\left.1, \ldots, 2^{K+1}\right\}=n b\left(H_{j^{\prime}}\right) \oplus n b s(H) \cup n b(H) \oplus n b s\left(H_{j^{\prime}}\right) \cup\left\{2^{K}, 2^{K}+1, \ldots, 2^{K+1}\right\}=$ $n b\left(H_{j^{\prime}} \cup H\right)\left\{2^{K}, 2^{K}+1, \ldots, 2^{K+1}\right\}$. If $j$ is labeled with a 1 , then $H_{j}=H_{j^{\prime}} \times H$ and $n b s\left(G_{j}\right)=n b s\left(G_{j^{\prime}}\right) \cup n b s(H)=n b s\left(H_{j^{\prime}}\right) \cup\left\{2^{K}, 2^{K}+1, \ldots, 2^{K+1} \Leftrightarrow 1\right\} \cup n b s(H)=$ $n b\left(H_{j^{\prime}} \times H\right) \cup\left\{2^{K}, 2^{K}+1, \ldots, 2^{K+1}\right\}$.

In particular, we have for the root $r$ of $T_{G}$ that $\left\{0,1,2, \ldots, 2^{K+1} \Leftrightarrow 1\right\}=$ $n b s\left(G_{r}\right)=n b s\left(H_{r}\right) \cup\left\{2^{K}, 2^{K}+1, \ldots, 2^{K+1} \Leftrightarrow 1\right\}$. Hence $n b\left(H_{r}\right) \geq 2^{K}$. So, if we remove all leaf-descendants of $i_{0}$ and $i_{1}$ from $G$, we remain with a graph with nimber at least $2^{K}$, hence with a graph with at least $s(K)$ vertices. As both $G_{i_{0}}$ and $G_{i_{1}}$ contain at least $s(K)$ vertices, $G$ contains at least $3 \cdot s(K)$ vertices. This ends the analysis of case 1 .

Case 2. $i_{2}$ has label 1. Then $G_{i_{2}}$ can be written as $G_{i_{2}}=\left(G_{i_{0}} \cup G_{j_{1}} \cup \ldots G_{j_{r}}\right) \times$ $\left(G_{i_{1}} \cup G_{k_{1}} \cup \ldots G_{k_{s}}\right)$. Write $H=G_{j_{1}} \cup \ldots G_{j_{r}}$, and $K=G_{k_{1}} \cup \ldots G_{k_{r}}$. Calculation shows that $n b s\left(G_{i_{2}}\right)=\left\{0,1,2, \ldots, 2^{K} \Leftrightarrow 1\right\} \cup 2^{K} \oplus n b s(H) \cup 2^{K} \oplus n b s(K)$. 
Consider the graph $G^{\prime}=G_{i_{0}} \cup(H \times K)$. There are two cases:

Case 2.1. $n b(H \times K)=2^{K}$. Then $G$ contains at least $3 \cdot s(K)$ vertices: $H$ and $K$ together contain at least $s(K)$ vertices and are disjoint from $G_{i_{0}}$ and $G_{i_{1}}$, which both also contain at least $s(K)$ vertices.

Case 2.2. $n b(H \times K)<2^{K}$. As none of the nodes $j_{1}, \ldots, j_{r}, k_{1}, \ldots, k_{s}$ is $K$ heavy, it follows that $n b s\left(G^{\prime}\right)=n b(H \times K) \oplus n b s\left(G_{i_{0}}\right) \cup n b\left(G_{i_{0}}\right) \oplus n b s(H \times K)=$ $n b(H \times K) \oplus\left\{0,1,2, \ldots, 2^{K} \Leftrightarrow 1\right\} \cup 2^{K} \oplus n b s(H) \cup 2^{K} \oplus n b s(K)=n b s\left(G_{i_{2}}\right)$. Now let $G^{\prime \prime}$ be the cograph, that corresponds to the cotree that is obtained by replacing in $T_{G}$ the subtree rooted at $i_{2}$ by the cotree $T_{G^{\prime}}$ of $G^{\prime}$. The nimberset for $i_{2}$ does not change under this replacement operation, and hence $n b\left(G^{\prime \prime}\right)=n b(G)$. But $G^{\prime \prime}$ has fewer vertices than $G$, contradiction. This ends the proof of case 2, and of theorem 6.5.

\section{Acknowledgements}

I like to thank Mark de Berg, Goos Kant, Ton Kloks, Dieter Kratsch, and Marc van Kreveld for several very helpful ideas and comments.

\section{References}

[1] E. R. Berlekamp, J. H. Conway, and R. K. Guy. Winning Ways for your mathematical plays, Volume 1: Games in General. Academic Press, New York, 1982.

[2] K. S. Booth and G. S. Lueker. Testing for the consecutive ones property, interval graphs, and graph planarity using $p q$-tree algorithms. J. Comp. Syst. Sc., 13:335-379, 1976.

[3] J. H. Conway. On Numbers and Games. Academic Press, London, 1976.

[4] D. G. Corneil, Y. Perl, and L. K. Stewart. A linear recognition algorithm for cographs. SIAM J. Comput., 4:926-934, 1985.

[5] M. Dietzfelbinger, A. Karlin, K. Mehlhorn, F. Meyer auf der Heide, H. Rohnert, and R. E. Tarjan. Dynamic perfect hashing: Upper and lower bounds. In Proceedings of the 29th Annual Symposium on Foundations of Computer Science, pages 524-531, 1988.

[6] M. C. Golumbic. Algorithmic Graph Theory and Perfect Graphs. Academic Press, New York, 1980.

[7] M. C. Golumbic, D. Rotem, and J. Urrutia. Comparability graphs and intersection graphs. Disc. Math., 43:37-46, 1983. 
[8] D. König. Theorie der endlichen und unendlichen Graphen. Akademische Verlagsgesellschaft, Leipzig, 1936.

[9] T. J. Schaefer. On the complexity of some two-person perfect-information games. J. Comp. Syst. Sc., 16:185-225, 1978.

[10] A. Tucker. An efficient test for circular-arc graphs. SIAM J. Comput., 9:124, 1980.

[11] P. van Emde Boas, R. Kaas, and E. Zijlstra. Design and implementation of an efficient priority queue. Mathematical Systems Theory, 10:99-127, 1984. 